\title{
Purinergic signaling microenvironments: An introduction
}

\author{
Erik M. Schwiebert • J. Gregory Fitz
}

Published online: 18 March 2008

(C) Springer Science + Business Media B.V. 2007

\begin{abstract}
The common theme of this introductory article and the minireviews that follow in this special issue is the concept of microenvironments within tissues and surrounding cells that would be ideal signaling venues for a biologically active purinergic ligand. Collectively, the editors/authors and the other contributing authors agree that nucleotides and nucleosides would be most potent within a confined system. A talented cadre of purinergics has been solicited to discuss purinergic signaling in his or her favorite microenvironment within a given organ or tissue. We are gratified by the large number of original articles that also have successfully navigated the peer review process and are part of this special issue. These
\end{abstract}

E. M. Schwiebert

Departments of Physiology and Biophysics and Cell Biology,

Gregory Fleming James Cystic Fibrosis Research Center,

Nephrology Research and Training Center, Recessive Polycystic

Kidney Disease Translational and Research CORE Centers,

University of Alabama at Birmingham,

Birmingham, AL, USA

J. G. Fitz

Department of Internal Medicine, University of Texas

Southwestern Medical Center,

Dallas, TX, USA

E. M. Schwiebert $(\square)$

University of Alabama at Birmingham,

MCLM 740, 1918, University Blvd.,

Birmingham, AL 35294-0005, USA

e-mail: eschwiebert@physiology.uab.edu

URL: www.discoverybiomed.com

Present address:

E. M. Schwiebert

DiscoveryBioMed, LLC, Innovation Depot,

1500 1st Avenue North,

Birmingham, AL 35203, USA concepts are not simply purinergic, but the idea of maximal potency in a tissue microenvironment and surrounding specialized cells within a tissue pertains to any autacoid or paracrine agonist.

Keywords Adenosine · ATP · Nucleosides · Nucleotides · Signaling

$\begin{array}{ll}\text { Abbreviations } & \\ \text { ATP } & \text { adenosine } 5^{\prime} \text { triphosphate } \\ \text { P2X or P2XR } & \text { P2X purinergic receptor channel } \\ \text { P2Y } & \text { P2Y G-protein-coupled receptor } \\ \text { ADP } & \text { adenosine diphosphate } \\ \text { AMP } & \text { adenosine monophosphate } \\ \text { CF } & \text { cystic fibrosis } \\ \text { ASL } & \text { airway surface liquid } \\ \text { ASF } & \text { airway surface fluid } \\ \text { PCL } & \text { periciliary liquid layer } \\ \text { ELF } & \text { epithelial lining fluid } \\ \text { Ca } & \text { Calcium } \\ \mathrm{Na}^{+} & \text {Sodium }\end{array}$

\section{Introduction}

Adenosine $5^{\prime}$ triphosphate (ATP) and adenosine are extracellular autocrine and paracrine signaling molecules. The most potent effects of ATP and adenosine are likely limited to extracellular microenvironments within tissues or immediately surrounding a cell or group of cells. These extracellular microenvironments may have a limited area of diffusion, have a small volume, have an ideal ionic composition and $\mathrm{pH}$, represent an unstirred layer near a membrane surface, represent a trapped interstitium, or any 
and all of the above. If $\mathrm{P} 1$ or $\mathrm{P} 2$ nucleotide receptors are present on a membrane that is exposed to released nucleotide or nucleoside in this microenvironment, these ligands can engage P1 G-protein-coupled receptors, P2Y Gprotein-coupled receptors or P2X receptor channels and affect the function of the cell on which it binds. Besides ATP and adenosine, other forms of nucleotides and nucleosides may also be active extracellular signaling ligands and may have unappreciated receptors. Inosine, thymidine, and even cyclic nucleotides are released by and may have effects on mammalian cells. The self-aggregation signal for the slime mold Dictyostelium discoideum, is cyclic adenosine monophosphate (AMP). Receptors for cyclic nucleotides have only been defined until now in the lower biological systems. Receptors for cyclic nucleotides as well as inosine or thymidine may also exist on mammalian cells and receive this autocrine signal within a microenvironment.

\section{Ideal microenvironments for purinergic signaling}

For simplicity, we focus on ATP and its metabolites. Many different microenvironments have been studied in great detail and are described and included below. One classical microenvironment that has emerged in importance in the cystic fibrosis (CF) research community is in an open system but is a stirred liquid layer trapped between the luminal membrane of the airways epithelium and the humidified air that fills the lung and airways. The airway surface liquid [ASL, also termed airway surface fluid (ASF), the periciliary liquid layer (PCL), and the epithelial lining fluid (ELF)] bathes motile respiratory cilia [1-4]. The humidified air above this layer and the ciliated apical or luminal membrane beneath this layer help close this microenvironment. Submucosal gland secretions may also contribute importantly to its composition. Ric Boucher and his many coworkers have presented many recent seminal contributions on the purinergic signaling that may occur in the ASL - among its many other properties $[3,4]$. There are similar microenvironments of this type that are also discussed in review articles within this special issue. Daniel Marcus and colleagues and Claire Mitchell and coworkers discuss liquid microenvironments in the ear and the eye, respectively $[5,6]$, which provide a medium for purinergic signaling.

A second classical microenvironment is a closed and open system that is compromised of a secretory gland or acinus and a collecting or draining duct. Iva Novak and colleagues have done seminal work in the pancreas in this regard $[7,8]$. Geoff Burnstock has often discussed the rich purinergic signaling that occurs in tubes or ducts $[9,10]$. In this microenvironment, glandular and acinar secretions may convey the purinergic ligands so that they may act in an autocrine manner at the acinus or in a paracrine manner within the duct. However, it is an open system in that these secretions eventually exit this tissue environment. The airway submucosal gland beneath the airway surface epithelium may be one such example that conveys purinergic signals to the surface where they may drive ciliary beat and other functions. The pancreatic acinus and duct, the sweat gland and duct, and the bile secretory apparatus and the bile duct are all important examples.

A third microenvironment is a closed system in which the purinergic ligands concentrate within a small volume and within a tissue. Here, the purinergic ligands bind and activate receptors until they are either degraded or reabsorbed by the cells that secreted them or by neighboring cells. The endolymph of the ear and the humors of the eye are ideal examples of such closed systems [11, 12]. Marcus and colleagues and Mitchell and coworkers discuss these systems in minireviews alluded to above $[5,6]$. Original articles and reviews in this issue and future issues of Purinergic Signalling speak to such systems in pancreatic islets and in the many renal tubules that comprise the nephron of the kidney [13]. In polycystic kidney and liver disease, tubules remodel into abnormal fluid-filled cysts. Multiple laboratories have argued that the fluid-filled lumen of the encapsulated cyst is a rich yet pathophysiological microenvironment for purinergic signaling [14-20]. The minireviews that follow this introductory article focus on specific microenvironments, cells and tissues that create and/or come in contact with such microenvironments, and all possible modes of purinergic signaling within these microenvironments.

\section{An ideal microenvironment paradigm for extracellular purinergic signaling: the synapse}

Many reviews in the past [21-23] and in the initial issues of this journal have documented well the roles of the P2X receptor channel at the synapse between neurons. Indeed, $\mathrm{P} 2 \mathrm{Y}$ receptors may play roles in synaptic transmission as well. This is analogous to the interplay between cholinergic and muscarinic receptor systems in cholinergic neurons and synapses in many different nuclei and regions of the brain. In fact, the purinergic field was born from the classical studies of extracellular ATP on the heart by Drury and Szent-Gyorgyi [24], in the definition of purinergic nerves in the enteric nervous system by Burnstock and colleagues [25], and on the release and effects of purinergic ligands such as ATP on skeletal muscle contractility by Tom Forrester (see "Historical Perspective" by T. Forrester in this issue, [26]). The authors lead this series of minireviews with the idea that the synapse is the ideal and, perhaps, the 
first or founding microenvironment for autocrine and paracrine purinergic biology.

The synapse is designed to have a limited diffusion distance between pre- and postsynaptic membranes where the principal ligand or neurotransmitter is reabsorbed by the presynapse or degraded in the synapse. For the sake of simplicity, our narrative here focused first on a purinergic synapse where released ATP acts as the principal ligand to evoke an action potential in the postsynaptic membrane. ATP as the lead neurotransmitter is released in response to an action potential in the presynaptic bulb that opens voltage-dependent calcium $\left(\mathrm{Ca}^{2+}\right)$ channels. This voltagedependent $\mathrm{Ca}^{2+}$ entry signal triggers the fusion or exocytosis of ATP-filled synaptic vesicles so that labile ATP is secreted into the synaptic cleft. Extracellular ATP-gated P2X receptor channels on the postsynaptic membrane have a large extracellular domain poised to bind that ATP immediately upon release within a synaptic microenvironment where there is a short diffusion distance and a small volume. In other words, the synapse is an ideal microenvironment for paracrine purinergic signaling. In this paradigm, the ATP is likely degraded to adenosine diphosphate (ADP), 5' AMP and adenosine, where adenosine may be recycled back into the presynapse by nucleoside transporters only to be a substrate to synthesize new ATP for metabolism or neurotransmission.

Several additional functions for P2X receptor channels have been proposed in neurotransmission driven by other neurotransmitters. For example, at the glutamate synapse, it is well known that ATP is released as a cotransmitter. A classical example of this is the hair-cell sound transduction process, where glutamate and ATP are coreleased to stimulate auditory afferents [11]. How may ATP affect responses to glutamate at glutamate, N-methyl-D-aspartate (NMDA), and kainate receptor channels in the postsynaptic membrane and/or at the presynapse in terms of release of ligand? Many possible cotransmitter effects have also been entertained and studied. We do not wish to debate the relative roles of P2X receptor channels in neurotransmission. The possible explanations of ATP effects at an excitatory glutamatergic synapse are given in simplistic terms. We only wish to show the synapse as a lead microenvironment to illustrate how and why local ATP signaling may be important.

First, the presence of a postsynaptic P2X receptor channel or channels would potentiate the excitation of glutamate postsynapse. Not only do NMDA, kainate, and glutamate itself potentiate $\mathrm{Ca}^{2+}$ and sodium $\left(\mathrm{Na}^{+}\right)$entry as $\mathrm{Ca}^{2+}$ permeable nonselective cation channels, but extracellular ATP-gated P2X receptor channels open a separate pathway for $\mathrm{Ca}^{2+}$ and $\mathrm{Na}^{+}$. Flow of $\mathrm{Ca}^{2+}$ and $\mathrm{Na}^{+}$through parallel pathways driven by these cotransmitters would lead to a much quicker development of a postsynaptic potential.
Moreover, with $\mathrm{Ca}^{2+}$ entering with $\mathrm{Na}^{+}$, it is likely that the action potential may be more long-lived and possibly more robust (i.e., having a shoulder or plateau due to $\mathrm{Ca}^{2+}$ entry beyond the $\mathrm{Na}^{+}$-driven spike). Second, ATP could potentiate glutamate release at the presynaptic neuron by potentiating $\mathrm{Ca}^{2+}$ entry driven by the preceding action potential. A more robust $\mathrm{Ca}^{2+}$ entry would cause more or secondary glutamate release and would keep the postsynaptic neuron firing. As for acetylcholine, ATP also has Gprotein-coupled receptors, the $\mathrm{P} 2 \mathrm{Y}$ receptors, which could modulate presynaptic and/or postsynaptic neuron function. Third, could metabolites of ATP limit activation of the postsynaptic and presynaptic neuron? One does not wish for the postsynaptic neuron to continue firing ad infinitum. One must have a limiting or negative feedback signal. Could this signal be adenosine? Not only does adenosine need to be recycled back into the neuron as a substrate for new ATP synthesis, but adenosine may also bind to P1 Gprotein-coupled receptors to have limiting effects or neuronal function. Of course, ATP release and signaling at an inhibitory synapse driven by $\gamma$-aminobutyric acid (GABA) or glycine would have different and modulating effects on inhibitory synapse. We do not discuss or illustrate these possible mechanisms because that is not the intent of this introductory minireview. Synaptic purinergic neurotransmission has been reviewed by Burnstock and others in many seminal reviews [9, 10, 21-23].

\section{Conclusions and future directions}

The founding concepts of purinergic signaling within tissues and tissue microenvironments were found in the heart, the enteric system (later to be duplicated in urinary bladder afferents, nociceptive neurons at the dorsal root ganglia of the spinal cord, etc.), and skeletal muscle by Forrester and coworkers [26]. This special issue of Purinergic Signalling focusing on the "Physiology of Nucleotide Release" is dedicated to these and other pioneers who would not give up the fight. All purinergics would agree that we owe the purinergic pioneers a great debt. Through their efforts, establishment of this journal, and the increasing volume of research in purinergic signaling, it is now accepted that ATP and its metabolites have two principal biological roles-as an extracellular signaling ligand and as an intracellular fuel.

Acknowledgments Work by EMS on purinergic signaling has been supported by past awards, DK54367 and HL63934, and is currently supported by DK67343. The PI also wishes to acknowledge the Cystic Fibrosis Foundation, the Polycystic Kidney Disease Research Foundation, and the American Heart Association for past support on the study of the "Physiology of Nucleotide Release." EMS is also a 
participant in the Gregory Fleming James Cystic Fibrosis Research Center, the Recessive PKD Translational and Research Core Centers, the Comprehensive Kidney Disease Research Center, and the Comprehensive Diabetes Center at UAB. Work by JGF has been long supported by NIH NIDDK R37 DK046082-14 and DK043278-18. JGF is the Donald Seldin Professor of Internal Medicine and the Chief of Internal Medicine at the University of Texas Southwestern Medical Center.

\section{References}

1. Schwiebert EM (2007) Extracellular autocrine nucleotide signaling in a microenvironment: integrative physiology in a minute volume of airway surface liquid. J Physiol 580(Pt. 2):359-360

2. Schwiebert EM, Zsembery A (2003) Extracellular ATP as a signaling molecule for epithelial cells. Biochim Biophys Acta 1615:7-32

3. Boucher RC (2007) Cystic fibrosis: a disease of vulnerability to airway surface dehydration. Trends Mol Med 13(6):231-240

4. Tarran R, Trout L, Donaldson SH, Boucher RC (2006) Soluble mediators, not cilia, determine airway surface liquid volume in normal and cystic fibrosis superficial airway epithelia. J Gen Physiol 127(5):591-604

5. Lee JH, Marcus D (2008) Purinergic signaling in the inner ear. DOI 10.1007/s11302-007-9089-4

6. Mitchell CH, Reigada D (2008) Purinergic signaling in the subretinal space: a role in the communication between the retina and the RPE. DOI 10.1007/s11302-007-9054-2

7. Novak I (2003) ATP as a signaling molecule: the exocrine focus. News Physiol Sci 18:12-17

8. Sorensen CE, Novak I (2001) Visualization of ATP release in pancreatic acini in response to cholinergic stimulus: use of fluorescent probes and confocal microscopy. J Biol Chem 276 (35):32925-32932

9. Burnstock G (2006) Purinergic signaling. Br J Pharmacol 147 (Suppl 1):S172-S181

10. Knight GE, Bodin P, De Groat WC, Burnstock G (2002) ATP is released from guinea pig ureter epithelium on distension. Am J Physiol 282(2):F281-F288

11. Housley GD et al (2002) Purinergic regulation of sound transduction and auditory neurotransmission. Audiol Neurootol 7 (1):55-61
12. Avila MY, Stone RA, Civan MM (2002) Knockout of A3 adenosine receptors reduces mouse intraocular pressure. Invest Ophthalamol Vis Sci 43(9):3021-3026

13. Hovater MB, Olteanu D, Welty EA, Schwiebert EM (2007) Purinergic signaling in the lumen of the normal nephron and in remodeled PKD encapsulated cysts. Purinergic Signal (this issue)

14. Olteanu D, Hovater MB, Schwiebert EM (2007) Intraluminal autocrine purinergic signaling within cysts: implications for the progression of diseases that involve encapsulated cysts. Am J Physiol 292:F11-F14

15. Schwiebert EM et al (2005) Extracellular ATP- and zinc-gated P2X receptor calcium entry channels: physiological sensors and therapeutic targets. Purinergic Signal 1(4):299-310

16. Wilson PD, Hovater JS, Casey CC, Fortenberry JA, Schwiebert EM (1999) ATP release mechanisms in primary cultures of epithelial cells derived from the cysts of polycystic kidneys. J Am Soc Neph 10:218-229

17. Schwiebert EM et al (2002) A detrimental autocrine and paracrine purinergic signaling loop exists in epithelial cells derived from polycystic kidneys. Am J Physiol 282(4):F763-F775

18. Turner CM, King BF, Srai KS, Unwin RJ (2007) Antagonism of endogenous putative $\mathrm{P} 2 \mathrm{Y}$ receptors reduces the growth of MDCK-derived cysts cultured in vitro. Am J Physiol 292(1): F15-F25

19. Unwin RJ, Bailey MA, Burnstock G (2003) Purinergic signaling along the renal tubule: the current state of play. News Physiol Sci $18: 237-241$

20. Doctor RB, Johnson S, Brodsky KS, Amura CR, Gattone V, Fitz JG (2007) Regulated ion transport in mouse liver cyst epithelial cells. Biochim Biophys Acta 1772(3):345-354

21. Burnstock G (2006) Historical review: ATP as a neurotransmitter. Trends Pharm Sci 27(3):166-176

22. Burnstock G (2006) Pathophysiology and therapeutic potential of purinergic signaling. Pharmacol Rev 58(1):58-86

23. Burnstock G (2007) Physiology and pathophysiology of purinergic neurotransmission. Physiol Rev 87(2):659-797

24. Drury AN, Szent-Gyorgyi A (1929) The physiological activity of adenine compounds with specific reference to their action upon the mammalian heart. J Physiol 68:213-237

25. Burnstock G, Campbell G, Satchell D, Smythe A (1970) Evidence that adenosine triphosphate or a related nucleotide is the transmitter substance released by non-adrenergic inhibitory nerves in the gut. Br J Pharmacol 40:668-688

26. Forrester T (2008) A case of serendipity. DOI 10.1007/s11302007-9090-y 